University of Nebraska - Lincoln

DigitalCommons@University of Nebraska - Lincoln

Paul Finkler Papers

Research Papers in Physics and Astronomy

7-1-1970

Limited Resurrection of the Born Approximation

Paul Finkler

University of Nebraska-Lincoln, pfinkler1@unl.edu

Follow this and additional works at: https://digitalcommons.unl.edu/physicsfinkler

Part of the Physics Commons

Finkler, Paul, "Limited Resurrection of the Born Approximation" (1970). Paul Finkler Papers. 6.

https://digitalcommons.unl.edu/physicsfinkler/6

This Article is brought to you for free and open access by the Research Papers in Physics and Astronomy at DigitalCommons@University of Nebraska - Lincoln. It has been accepted for inclusion in Paul Finkler Papers by an authorized administrator of DigitalCommons@University of Nebraska - Lincoln. 


\title{
Limited Resurrection of the Born Approximation
}

\author{
Paul Finkler \\ Behlen Laboratory of Physics, University of Nebraska, Lincoln, Nebraska 68508
}

(Received 25 February 1970)

\begin{abstract}
It is shown that the ordinary Born approximation for $p n$ and $p p$ charge-exchange scattering correctly accounts for (1) the shape of the forward peak for $0 \leq(-t) \leq \mu^{2} / 2$ at $P_{L}=8 \mathrm{GeV} / c$, and (2) the energy dependence of the cross sections at $t=0$ in the energy range $P_{L}=2-8 \mathrm{GeV} / c$. This result is analogous to the well-known success of the electric Born approximation in $\pi^{+}$photoproduction. It is then shown that the simplest interpretation of this surprising result within the framework of Regge-pole theory is in terms of the fixed poles which are allowed by unitarity in hadronic amplitudes at certain nonsense points of right signature. Finally, it is shown how such a fixed pole at a nonsense point of one helicity amplitude affects another amplitude for which the corresponding point is sense.
\end{abstract}

\section{INTRODUCTION}

$T$ O date, no simple and esthetically pleasing explanation has been found for the sharp forward $(-t \approx 0)$ peaks at high energy $(s \rightarrow \infty)$ in chargedpion photoproduction ${ }^{1}$ and nucleon-nucleon chargeexchange scattering. ${ }^{2}$ It is common belief that the effect is due in some way to the pole in the scattering amplitude at $t=\mu^{2}$, which is associated with chargedpion exchange. The difficulty with this explanation is that the amplitude for pion exchange has the form $t /\left(t-\mu^{2}\right)$. The factor $t$, which makes the pion contribution vanish in the forward direction, is ultimately due to the pseudoscalar nature of the pion-i.e., to the fact that the pion has unnatural parity.

Attempts to remove or alter the disastrous factor $t$ have utilized absorption corrections ${ }^{3}$ or pion conspiracy. ${ }^{4}$ Absorptionlike effects can be introduced into Regge theory by invoking Regge cuts. ${ }^{5}$ We will not comment further on these techniques except to note that, in practice, they necessitate the introduction of a number of additional parameters beyond those which are normally present in a particle-exchange or Regge-pole model.

It is amusing to note that when Boyarski et al..$^{1}$ first presented their photoproduction data, they observed that it was in excellent agreement with the predictions of the electric Born approximation (Fig. 1), at least for $0<-t \lesssim \mu^{2}$. The underlying significance behind this success of the Born approximation has been obscured by two features peculiar to photoproduction and other photon processes: the requirements of gauge invariance and the possibility of weak fixed poles.

It was originally thought that Reggeization of the pion contribution might be complicated by gauge invariance, which requires that the two diagrams of

${ }^{1}$ A. M. Boyarski, F. Bulos, W. Busza, R. Diebold, S. D. Ecklund, G. E. Fischer, J. Rees, and B. Richter, Phys. Rev. Letters 20, 300 (1968).

${ }^{2}$ G. Manning, A. G. Parham, J. D. Jafar, H. B. vanderRaay, D. H. Reading, D. G. Ryan, B. D. Jones, J. Malos, and N. H. Lipman, Nuovo Cimento 41, 167 (1966).

${ }^{3}$ L. Durand, Phys. Rev. Letters 19, 1345 (1967).

${ }^{4}$ J. S. Ball, W. R. Frazer, and M. Jacob, Phys. Rev. Letters 20, 518 (1968); R. J. N. Phillips, Nucl. Phys. B2, 394 (1967).

${ }^{5}$ L. Van Hove, Phys. Letters 24B, 183 (1967).
Fig. 2 must be considered together. It has since been shown, however, that the Reggeized version of Fig. 2(a) is separately consistent with gauge invariance. ${ }^{6}$

The other obfuscation arises because photoproduction is a "weak" process, i.e., it is usually treated to first order in the charge (or magnetic moments). This has led to a folklore of "weak fixed poles." The argument given for these weak fixed poles is that, to lowest order in the charge $e$, the weak amplitude never appears bilinearly in the unitarity condition. Hence, such fixed poles will not conflict with unitarity. This argument is always qualified by the statement that these poles would be ordinary moving poles if electromagnetic effects were treated to all orders in $e$ but in lowest order the trajectories condense onto nonsense points. The amplitude for Fig. 2(b) gives rise to a fixed pole at the right-signature nonsense point $J=0$, and it is this amplitude which is responsible for the success of the Born approximation at $t=0$.

It has been shown, however, that the arguments for weak fixed poles, if carried one step further, would lead to weak poles which contribute strongly to strong processes. ${ }^{8}$ This peculiar and unacceptable result can be obtained by considering the implications of factorizing the residue of the weak pole before going to lowest order in $e$.

The main purpose of this paper is to point out that the Born approximation for nucleon-nucleon chargeexchange scattering also is in excellent agreement with experiment in the region of the sharp spike near $t=0$. In this case, there is neither gauge invariance nor weak poles to cloud the issue. Whatever is responsible for this surprising success of the Born approximation must be a property of hadronic amplitudes.

Our second purpose is to show that the Born contribution to $N N$ charge exchange can be interpreted simply and consistently in terms of right-signature nonsense fixed poles. Such fixed poles are allowed by unitarity under certain conditions and these conditions are satisfied in the present example. ${ }^{8}$ We will examine

${ }^{6}$ J. S. Ball and M. Jacob, Nuovo Cimento 54, 620 (1968).

7 M. Halpern, Phys. Rev. 160, 1441 (1967); L. Jones and C. Rebbi, Report No. CALT 68-168 1968 (unpublished).

${ }^{8}$ P. Finkler, Phys. Rev. D 1, 1172 (1970). 


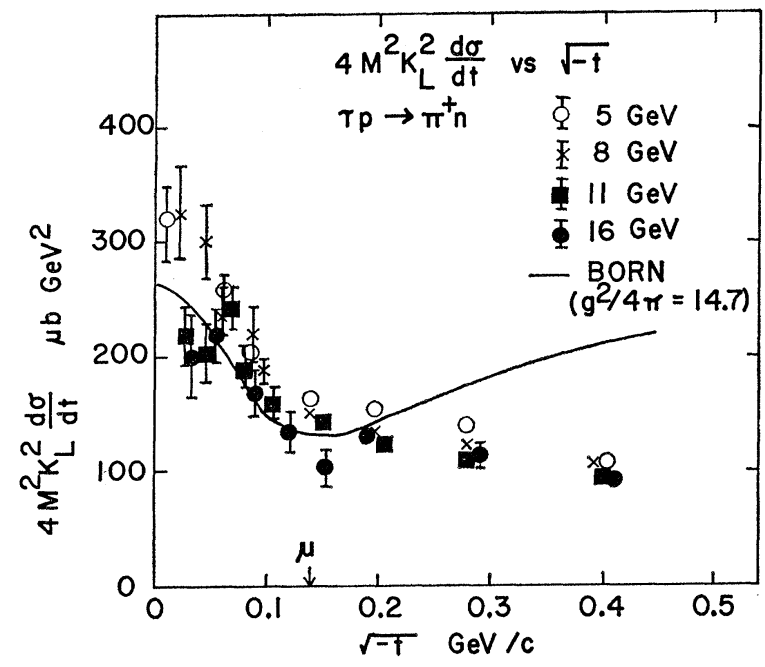

FIG. 1. Comparison of the electric Born approximation with the $\pi^{+}$photoproduction data from 5 to $16 \mathrm{GeV}$ (after Boyarski et al., Ref. 1).

the amplitudes to illustrate another point; namely, that a fixed pole at a nonsense point in one helicity amplitude contributes as well to the asymptotic behavior of another helicity amplitude in which the corresponding point is sense.

\section{BORN APPROXIMATION COMPARED WITH EXPERIMENT}

We will rely heavily on the $N N$ scattering formalism of Goldberger, Grisaru, MacDowell, and Wong. ${ }^{9}$ The differential cross section for unpolarized nucleons is given in terms of $t$-channel helicity amplitudes $\phi_{i}{ }^{t}$, $i=1, \ldots, 5$ by $^{10}$

$\frac{d \sigma}{d t}=4\left[s\left(s-4 M^{2}\right)\right]^{-1}\left(\frac{1}{2} \pi\right)\left(\sum_{i=1}^{4}\left|\phi_{i}{ }^{t}\right|^{2}+4\left|\phi_{5}{ }^{t}\right|^{2}\right)$,

where $4\left[s\left(s-4 M^{2}\right)\right]^{-1}=\left(M P_{L}\right)^{-2}=0.4423 \mathrm{mb} / P_{L}{ }^{2}$ and

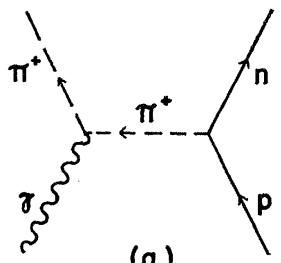

(a.)

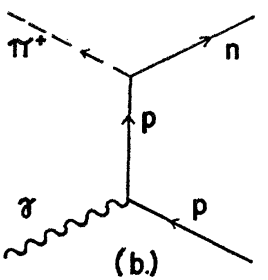

(b.)
FIG. 2. Feynman diagrams for the electric Born approximation in $\pi^{+}$photoproduction.

${ }^{9}$ M. L. Goldberger, U. T. Grisaru, S. W. MacDowell, and D. Y. Wong, Phys. Rev. 120, 2250 (1960), hereafter referred to as GGMW.

${ }^{10} \mathrm{We}$ are using the formalism of "channel II" (i.e., $N \bar{N} \rightarrow N \bar{N}$ ) in GGMW, but we have dropped the bars from their amplitudes $\bar{f}_{i}$ and $\bar{G}_{i}$. Furthermore, our $\phi_{i}{ }^{t}$ are related to the $\phi_{i}$ of GGMW by $\phi_{i}{ }^{t}=2 E \phi_{i}$. Since we wish $s$ to be the energy variable for $N N \rightarrow N N$, we use Eq. (A2) of Ref. 9 with the replacements $(s, \bar{t}, t)_{\mathrm{GG} \mathrm{MW}} \rightarrow(t, s, u)$.
$P_{L}$ is the laboratory momentum of the incident nucleon. The connection between the helicity amplitudes $\phi_{i}{ }^{t}$ for $N \bar{N} \rightarrow N \bar{N}$ and the amplitudes $G_{i}$ which satisfy the Mandelstam representation is

$$
\begin{aligned}
& f_{1}=\frac{1}{2}\left(\phi_{1}{ }^{t}-\phi_{2}{ }^{t}\right)=E^{2} G_{1}-z p^{2} G_{2}+M^{2} G_{3}, \\
& f_{2}=\frac{1}{2}\left(\phi_{1}{ }^{t}+\phi_{2}{ }^{t}\right)=\left(E^{2} G_{2}+M^{2} G_{4}\right) z-p^{2} G_{5}, \\
& f_{3}=\frac{1}{2}\left[\phi_{3}{ }^{t} /(1+z)-\phi_{4}{ }^{t} /(1-z)\right]=-p^{2} G_{3}, \\
& f_{4}=\frac{1}{2}\left[\phi_{3}{ }^{t} /(1+z)+\phi_{4}{ }^{t} /(1-z)\right]=M^{2} G_{2}+E^{2} G_{4}, \\
& f_{5}=\phi_{5}{ }^{t} M /\left[E\left(1-z^{2}\right)^{1 / 2}\right]=-M^{2}\left(G_{2}+G_{4}\right),
\end{aligned}
$$

where $p, E$, and $z$ are related to the Mandelstam variables by $t=4 E^{2}=4\left(p^{2}+M^{2}\right), \quad u=-2 p^{2}(1-z)$, and $s=-2 p^{2}(1+z)$.

For the processes we are considering, the $s, t$, and $u$ channels correspond to $p n \rightarrow n p, p \bar{n} \rightarrow \bar{n} p$, and $p \bar{p} \rightarrow n \bar{n}$, respectively. The $t$ channel is thus pure $I=1$. The Born terms are given in GGMW to be

$$
t \text {-channel } \pi^{+} \text {exchange: }
$$

$$
G_{1}=4\left(g^{2} / 4 \pi\right)\left(\mu^{2}-t\right)^{-1}, \quad G_{2}=G_{3}=G_{4}=G_{5}=0,
$$

$u$-channel $\pi^{0}$ exchange:

$$
G_{1}=G_{2}=-G_{3}=-G_{4}=G_{5}=\frac{1}{2}\left(g^{2} / 4 \pi\right)\left(\mu^{2}-u\right)^{-1},
$$

where $\mu^{2}=0.0195$ is the pion mass squared and $g^{2} / 4 \pi$ $=14.8 \pm 0.3$ is the rationalized and renormalized pionnucleon coupling constant. ${ }^{11}$

In addition to the pion poles, the Born approximation includes a contribution from the deuteron pole in the $s$ channel. This contribution is expected to be small. The Feynman amplitude for the deuteron ${ }^{12}$ quoted in GGMW, however, has an $s$ dependence which exceeds the established bounds on scattering amplitudes. ${ }^{13}$ This difficulty can be traced to the $D$-wave component of the deuteron amplitude. The momentum factors in the Feynman amplitude which guarantee correct threshold behavior for this component are responsible for the anomalous asymptotic behavior. At the position of the deuteron pole, however, the $D$-wave component amounts to only a few percent of the $S$-wave component. In order to obtain a realistic estimate of the deuteron contribution at high energy, we set the $D$-wave/ $S$-wave ratio equal to zero. In this approximation, the effect of the deuteron is less than $3 \%$ of that of the pions above $P_{L}=3 \mathrm{GeV} / c$. Consequently, we omitted the deuteron from further consideration.

11 V. K. Samaranayake and W. S. Woolcock, Phys. Rev. Letters 15,936 (1965). These authors find $f^{2}=0.0822 \pm 0.0018$, which leads to the quoted value of $g^{2} / 4 \pi$.

12 R. Blankenbecler, M. L. Goldberger, and F. R. Halpern, Nucl. Phys. 12, 629 (1959).

${ }^{13}$ See, for example, the fixed angle bounds summarized by R. J. Eden in High Energy Collisions of Elementary Particles, (Cambridge U. P., Cambridge, England, 1967). Also, compare the comments in the last paragraph of Sec. IV of GGMW with their Eq. (A3). 
The contribution of the pions to the helicity amplitudes is given by

$$
t \text {-channel } \pi^{+} \text {: }
$$$$
\phi_{1}^{t}=-\phi_{2}{ }^{t}=-\left(g^{2} / 4 \pi\right)\left[-t /\left(\mu^{2}-t\right)\right],
$$

$u$-channel $\pi^{0}$ :

$$
\phi_{3}{ }^{t}=\phi_{4}{ }^{t}=\phi_{5}{ }^{t}=0
$$

$$
\begin{aligned}
\phi_{1}{ }^{t}=\phi_{3}{ }^{t}=\phi_{5}{ }^{t}=0, \phi_{2}{ }^{t}=\phi_{4}{ }^{t}=-\frac{1}{2}\left(g^{2} / 4 \pi\right)[ & \left.-u /\left(\mu^{2}-u\right)\right] \\
& \approx-\frac{1}{2}\left(g^{2} / 4 \pi\right),
\end{aligned}
$$

where, in the last expression, we used $-u /\left(\mu^{2}-u\right) \cong 1$ for small $t$ and large $s(p n \rightarrow n p)$ or large $u(p \bar{p} \rightarrow n \bar{n})$. With this slight approximation, the differential cross section can be expressed simply as

$$
\begin{aligned}
\frac{d \sigma}{d t}=\frac{1}{4} \pi\left(M P_{L}\right)^{2}\left(\frac{g^{2}}{4 \pi}\right)^{2} & \left(1+X^{2}-X\right) \\
& =\left(76.6 \mathrm{mb} / P_{L^{2}}\right)\left(1+X^{2}-X\right),
\end{aligned}
$$

where $X=-2 t /\left(\mu^{2}-t\right)$.

This result is compared in Fig. 3 with the differential cross section for $p n \rightarrow n p$ at $P_{L}=8 \mathrm{GeV} / c$ observed by Manning et al. ${ }^{2}$ The error bars on the data shown in Fig. 3 reflect relative errors of $5-10 \%$ and do not include an over-all systematic uncertainty of $30 \%{ }^{2}$ To aid the reader's eye we have indicated in the figure the effect of raising the data by this amount. The agreement of the Born approximation with the data near $t=0$ is remarkably good. This agreement is even more remarkable in that no free parameters have been used. Furthermore, the energy dependence of the forward cross section for $p n \rightarrow n p$ and for $p \bar{p} \rightarrow n \bar{n}$ predicted by the Born approximation, $d \sigma / d t(t=0)=76.6 \mathrm{mb} / P_{L}{ }^{2}$,

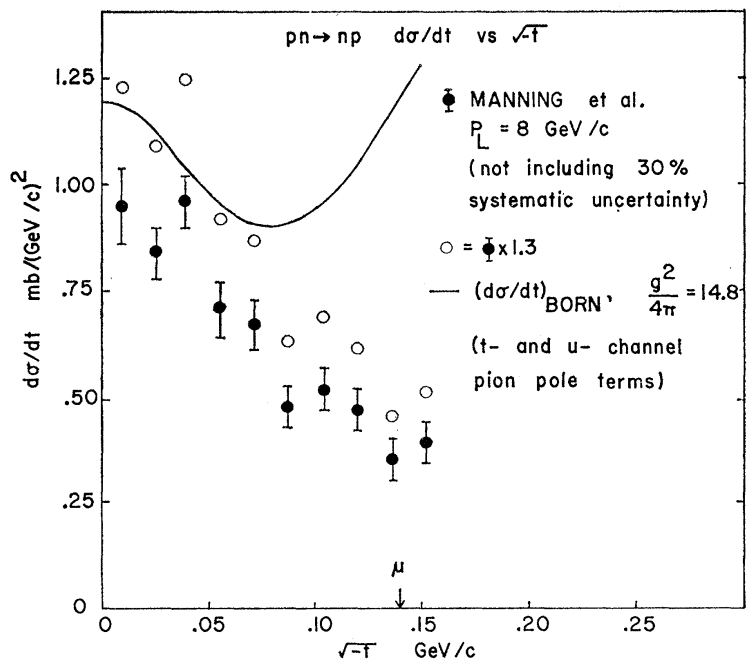

FIG. 3. Comparison of the Born approximation with the $p n$ chargeexchange data at $P_{L}=8 \mathrm{GeV} / c$ (Manning et al., Ref. 2).

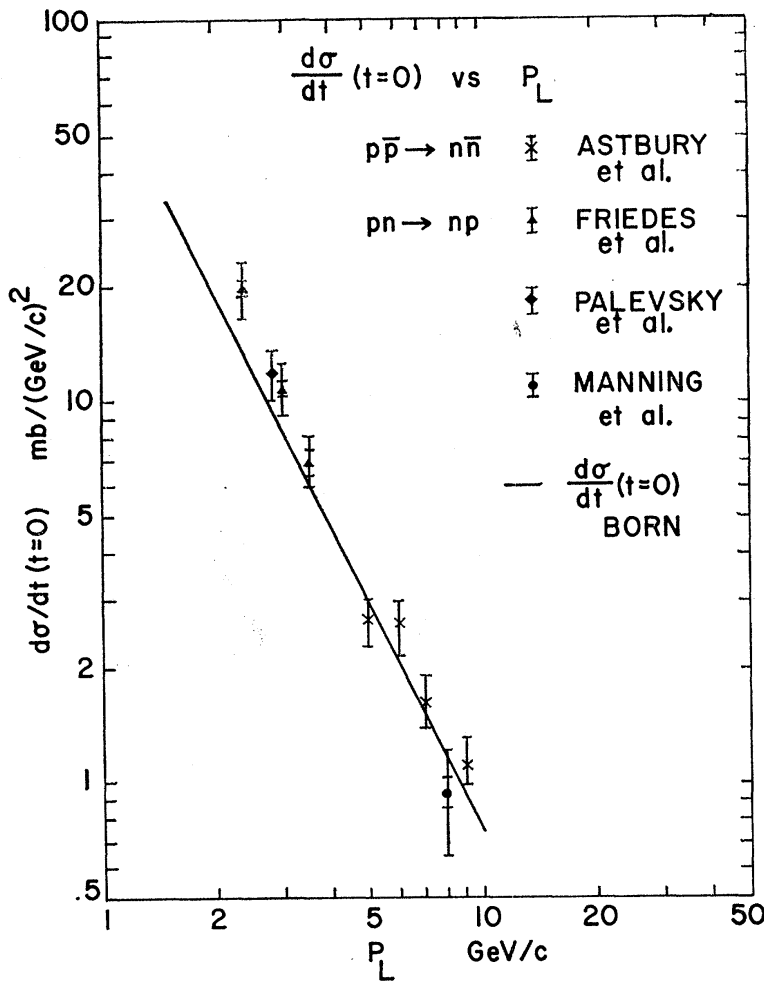

FIG. 4. Comparison of the Born approximation with the $p n$ and $p \vec{p}$ charge-exchange data at $t=0$. The error bars on the $p n \rightarrow n p$ data reflect the systematic uncertainties reported in Ref. 2(Manning et al.) and Ref. 15 (Friedes et al. and Palevsky et al. $)$. The $p \bar{p} \rightarrow n \bar{n}$ data are the extrapolations to $t=0$ reported in Ref. 14 (Astbury et al.) which already reflect systematic uncertainties.

is also in good agreement with experiment above 2 $\mathrm{GeV} / c, 2,14,15$ as seen in Fig. 4.

The unexpected feature of our result is that, at $t=0$, the sole contribution to the charge-exchange cross section comes from the $u$-channel (neutral) pionexchange amplitude.

The predictions of Eq. (4) disagree violently with the data in Fig. 3 for $(-t)>\frac{1}{2} \mu^{2}$, primarily because of the growth of the $t$-channel pion Born term. In Fig. 1 we see that an analogous disagreement occurs in the photoproduction case for $(-t)>\mu^{2}$. The resolution of this difficulty can be found readily in a Regge theory with evasive trajectories. In such a theory the $t$-channel pion contribution, which we have treated in particleexchange fashion, will be replaced by its Regge form. The pion trajectory lies slightly below $\alpha=0$ in the region of interest and its Regge residue function can be

${ }^{14} p \bar{p} \rightarrow n \bar{n}$ data: P. Astbury, G. Brautti, G. Finocchiaro, A. Michelini, D. Websdale, C. H. West, E. Polgar, W. Beusch, W. E. Fischer, B. Gobbi, and M. Pepin, Phys. Letters 23, 160 (1966).

${ }_{15} p n \rightarrow n p$ data: J. L. Friedes, H. Palevsky, R. L. Stearns, and R. J. Sutter, Phys. Rev. Letters 15, 38 (1965); H. Palevsky, J. A. Moore, R. L. Stearns, H. R. Menther, R. J. Sutter, R. E. Chrien, A. P. Jain, and K. Otnes, ibid.9, 509 (1962). 
expected to differ somewhat from that given by Eq. (3), apart from the factor $t$. Other trajectories known to be important here are those of $\rho$ and $A_{2}$, which lie above that of the pion. These trajectories give contributions to the amplitude $\left(\phi_{1}{ }^{t}+\phi_{2}{ }^{t}\right)$ which are finite at $t=0$, but small in this energy region, ${ }^{16}$ as well as contributions to other amplitudes which vanish at $t=0$. Thus, nearly all of the contributions of evasive trajectories vanish at $t=0,{ }^{17}$ exposing the $u$-channel pion Born contribution. The observed decrease in the cross section away from $t=0$ is the result of destructive interference between the $u$-channel pion term and the growing $\pi$ and $A_{2}$ Regge terms. The $\rho$ Regge term is responsible for the difference in the cross sections for $p n \rightarrow n p$ and $p \bar{p} \rightarrow n \bar{n}$.

We have made no attempt to fit the data for $(-t) \geq \frac{1}{2} \mu^{2}$ by utilizing these trajectories. Introducing the necessary parameters would only detract from the simplicity of our result-namely, the success of the Born approximation for $(-t) \simeq 0$.

The question remains as to how the $u$-channel pion Born term itself fits into Regge theory. The contributions of this term to the various helicity amplitudes have integer power behavior in $s$ ( $s^{0}$ for the leading term in the present case). This is just the behavior associated with fixed Regge poles. If fixed poles were forbidden, then, necessarily, there would be other contributions (e.g., resonances) to the amplitudes which would combine with the $u$-channel pion Born term, in the sense of Dolen-Horn-Schmid duality, ${ }^{18}$ to form one or more ordinary trajectories. If fixed poles were allowed, however, then the Born term could contribute to these poles and the integer power behavior associated with the Born term could persist asymptotically. As we will see in Sec. III, the possibility of interpreting the $u$-channel pion Born term as right-signature, nonsense fixed poles in the $t$-channel partial-wave amplitudes follows in a natural way from the Froissart-Gribov projection of that term. Furthermore, this possibility is perfectly consistent with the idea of DHS duality, provided that the fixed poles are allowed.

That $u$ - or $s$-channel effects can reflect themselves as $t$-channel fixed poles is an appealing prospect for Regge theory. Since these effects need not be associated with definite $t$-channel quantum numbers, they automatically "conspire." With the conspiracy thus accounted for by fixed poles, one can now treat all ordinary trajectories, including the pion, on the same evasive footing.

\footnotetext{
${ }^{16} \mathrm{It}$ should be noted that at asymptotic energies, it is this contribution of the $\rho$ and $A_{2}$ which will dominate the cross section at $t=0$.

17 In the photoproduction case, all contributions of evasive trajectories vanish at $t=0$. At asymptotic energies the photoproduction cross section should exhibit a forward dip to the Bornapproximation value.

${ }_{18}$ R. Dolen, D. Horn, and C. Schmid, Phys. Rev. 166, 1768 (1968), hereafter referred to as DHS.
}

\section{FIXED POLES AND BORN APPROXIMATION}

It was shown recently that nonsense fixed poles of right signature are often allowed by unitarity. ${ }^{8}$ Indeed, it happens that such fixed poles are forbidden by unitarity only when the initial or final state of a reaction is a two-body state having the lowest threshold associated with a given set of quantum numbers and when the spins of the particles in that state are sufficiently small. These fixed poles have nothing to do with moving cuts in the $J$ plane. Instead, they owe their existence to the fixed cuts which appear explicitly in the definition of the continued partial-wave amplitudes. The conditions, from which one can determine whether or not a fixed pole is allowed at a particular nonsense point in a particular amplitude, are given in Ref. 8.

The conditions which allow fixed poles are always satisfied at nonsense points of the $N \bar{N}$ scattering amplitudes. The reason that fixed poles are allowed here is that there are many-body states below the $N \bar{N}$ threshold. As we will see, the $u$-channel pion pole contribution is perfectly compatible with an interpretation in terms of fixed poles. There is a feature of the $N \bar{N}$ amplitudes, however, which makes it worth our while to examine in detail just how this compatibility comes about.

The feature in question is a rather general property of amplitudes for the scattering of spinning particles and relates to the nature of the subtractions in the fixed- $t$ dispersion relations satisfied by helicity amplitudes. This matter has already been examined by Mandelstam in the context of pion-nucleon scattering, ${ }^{19}$ but it acquires new relevance now that fixed poles are known to be allowed.

The partial-wave expansion of the definite parity amplitudes $f_{i}$ of Eq. (1) is given by ${ }^{20}$

$$
\begin{aligned}
& f_{1}(t, z)=\sum_{J=0}(2 J+1) h_{0}^{J}(t) P_{J}(z) \\
& f_{2}(t, z)=\sum_{J=0}(2 J+1) h_{11}{ }^{J}(t) P_{J}(z), \\
& f_{3}(t, z)=\sum_{J=1}(2 J+1)\left[h_{1}^{J}(t)\left(z P_{J^{\prime}}\right)^{\prime}-h_{22}{ }^{J}(t) P_{J^{\prime \prime}}\right] / \\
& \quad[2 J(J+1)] \\
& f_{4}(t, z)=\sum_{J=1}(2 J+1)\left[h_{22}{ }^{J}(t)\left(z P_{J^{\prime}}\right)^{\prime}-h_{1}^{J}(t) P_{J^{\prime \prime}}\right] / \\
& \quad[2 J(J+1)], \\
& f_{5}(t, z)=\sum_{J=1}(2 J+1)\left[-2 h_{12}^{J}(t) P_{J^{\prime}}\right] /[2 J(J+1)]^{1 / 2} .
\end{aligned}
$$

These equations can be inverted so as to express the partial-wave amplitudes directly as projections of the

19 S. Mandelstam, Nuovo Cimento 30, 1113 (1963).

${ }^{20}$ I. J. Muzinich, Phys. Rev. 130, 1571 (1963). 
invariant amplitudes $G_{i}{ }^{20}$ :

$$
\begin{gathered}
h_{0}{ }^{J}(t)=E^{2} g_{1}{ }^{J}-p^{2}\left[J g_{2}{ }^{J-1}+(J+1) g_{2}{ }^{J+1}\right] / \\
(2 J+1)+M^{2} g_{3}{ }^{J}, \\
h_{11}{ }^{J}(t)=E^{2}\left[J g_{2}{ }^{J-1}+(J+1) g_{2}{ }^{J+1}\right] /(2 J+1) \\
+M^{2}\left[J g_{4}{ }^{J-1}+(J+1) g_{4}{ }^{J+1}\right] / \\
(2 J+1)-p^{2} g_{5}{ }^{J}, \\
h_{22}{ }^{J}(t)=-p^{2} g_{3}{ }^{J}+M^{2}\left[(J+1) g_{2}{ }^{J-1}+J g_{2}{ }^{J+1}\right] /(2 J+1) \\
+E^{2}\left[(J+1) g_{4}{ }^{J-1}+J g_{4}{ }^{J+1}\right] /(2 J+1), \\
h_{1}{ }^{J}(t)=-p^{2}\left[(J+1) g_{3}{ }^{J-1}+J g_{3}{ }^{J+1}\right] /(2 J+1) \\
+M^{2} g_{2}{ }^{J}+E^{2} g_{4}{ }^{J}, \\
h_{12}{ }^{J}(t)=M^{2}[J(J+1)]^{1 / 2}[2(2 J+1)]^{-1} \\
\times\left[g_{2}{ }^{J+1}-g_{2}{ }^{J-1}+g_{4}{ }^{J+1}-g_{4}{ }^{J-1}\right] .
\end{gathered}
$$

In the last equation the quantities $g_{i}{ }^{J}$ are given by

$g_{i}{ }^{J}(t)=\frac{1}{2} \int_{-1}^{1} d f P_{J}(z) G_{i}(t, z), \quad j=1,2, \ldots, 5$.

The amplitudes $G_{i}$ satisfy a Mandelstam representation with no arbitrary subtractions. We assume that $t$ is so chosen that the $G_{i}$ satisfy fixed $t$ dispersion relations with no subtractions:

$G_{i}(t, z)=\frac{1}{\pi} \int_{u_{0}} \frac{G_{i}{ }^{(u)}\left(t, u^{\prime}\right) d u^{\prime}}{u^{\prime}-u}+\frac{1}{\pi} \int_{s_{0}} \frac{G_{i}^{(s)}\left(t, s^{\prime}\right) d s^{\prime}}{s^{\prime}-s}$.

The lower limits of integration are chosen low enough to include the $u$ - and $s$-channel Born terms. As usual, one now can define the analytically continuable partialwave amplitudes of definite signature $h_{\lambda}^{ \pm}(J, t)$, which bear the same relation to the quantities

$$
\begin{array}{r}
g_{i}^{ \pm}(J, t)=\left(2 \pi p^{2}\right)^{-1} \int_{u_{0}, s_{0}}\left[G_{i}(u)\left(t, u^{\prime}\right) \pm G_{i}{ }^{(s)}\left(t, u^{\prime}\right)\right] \\
\times Q_{J}\left(1+\frac{u^{\prime}}{2 p^{2}}\right) d u^{\prime}
\end{array}
$$

as the $h_{\lambda}{ }^{J}(t)$ do to the $g_{i}{ }^{J}(t)$. Hereafter we will not specify the signature superscript.

From Eq. (5) one immediately can read off the energy dependence of the contribution of a Regge pole to the $f_{i}$. A pole at $J=\alpha$ in $h_{0}(J)\left[h_{11}(J)\right]$ contributes a term $s^{\alpha}$ to $f_{1}\left[f_{2}\right]$, one at $J=\alpha$ in $h_{12}$ contributes $s^{\alpha-1}$ to $f_{5}$, whereas a pole in $h_{1}(J)\left[h_{22}(J)\right]$ contributes a term $s^{\alpha-1}$ to $f_{3}\left[f_{4}\right]$ and a term $s^{\alpha-2}$ to $f_{4}\left[f_{3}\right]$. In addition, the point $J=0$ is a sense-sense point for $h_{0}$ and $h_{11}$, a nonsense-nonsense point for $h_{1}$ and $h_{22}$, and a nonsensesense point for $h_{12}$.

When one examines the leading contribution of the $u$-channel pion Born term to the various $f_{i}$, one finds that is contributes a term $s^{0}$ to $f_{1}$ and $f_{2}$, and a term $s^{-1}$ to $f_{3}$ and $f_{4}$ (there happens to be no contribution to $f_{5}$ ). The Born contributions to $f_{3}$ and $f_{4}$ can be interpreted readily as right-signature nonsense fixed poles in $h_{1}(J)$ and $h_{22}(J)$. The feature which requires clarification is the Born contribution to $f_{1}$ and $f_{2}$ at $J=0$, which is a sense point for $h_{0}(J)$ and $h_{11}(J)$.

What we wish to emphasize is that these contributions have nothing to do with Kronecker deltas in $J$. Rather, they are merely subsidiary contributions of the perfectly analytic nonsense fixed poles in the other amplitudes. In order to establish this point, let us examine the analytically continued version of Eq. (6) near $J=0$, which is a nonsense point for $h_{22}, h_{1}$, and $h_{12}$. Keeping only the terms which are singular at $J=0$, we have

$$
\begin{aligned}
h_{22}(J, t) \cong M^{2} g_{2}(J-1, t)+E^{2} g_{4}(J-1, t), \\
h_{1}(J, t) \cong-p^{2} g_{3}(J-1, t), \\
h_{12}(J, t) \cong-\frac{1}{2} M^{2} J^{1 / 2}\left[g_{2}(J-1, t)+g_{4}(J-1, t)\right] .
\end{aligned}
$$

The quantities $g_{i}(J-1, t), i=2,3,4$ each have a pole at $J=0$ which arises from the Legendre function of the second kind in Eq. (9). The $u$-channel pion contribution to these poles is obtained by using $G_{2,3,4}{ }^{(u)}\left(t, u^{\prime}\right)$ $=(+,-,-) \frac{1}{2}\left(g^{2} / 4 \pi\right) \delta\left(u^{\prime}-\mu^{2}\right)$ in Eq. (9), as dictated by Eq. (2).

Clearly, the poles of $g_{2,3,4}(J-1, t)$ at $J=0$ are in oneto-one correspondence with the nonsense fixed poles in $h_{22}(J, t), h_{1}(J, t)$ and $J^{-1 / 2} h_{12}(J, t)$ (although the $u$-channel pion contributes oppositely to $g_{2}$ and $g_{4}$ and so does not contribute to $h_{12}$ ). These same $g_{i}$ contribute to the following differences between the continued and physical partial-wave amplitudes for which $J=0$ is a sense point:

$$
\begin{gathered}
h_{0}(0, t)-h_{0}{ }^{0}(t)=\left\{-p^{2} J\left[g_{2}(J-1, t)-g_{2}{ }^{J-1}(t)\right]\right\}_{J=0} \\
\quad=-\left.p^{2}\left[J g_{2}(J-1, t)\right]\right|_{J=0}, \\
h_{11}(0, t)-h_{11}{ }^{0}(t) \quad \\
=\left\{E^{2} J\left[g_{2}(J-1, t)-g_{2}{ }^{J-1}(t)\right]\right. \\
\left.\quad+M^{2} J\left[g_{4}(J-1, t)-g_{4}{ }^{J-1}(t)\right]\right\}_{J=0} \\
= \\
\left.\quad\left[E^{2} J g_{2}(J-1, t)+M^{2} J g(J-1, t)\right]\right|_{J=0},
\end{gathered}
$$

where we have used the fact that $J g_{i}{ }^{J-1}(t)=0$ at $J=0$. Since these differences derive solely from the nonsense poles associated with $h_{22}, h_{1}$, and $h_{12}$, they can always be obtained by analytically continuing these three amplitudes to their nonsense points. Furthermore, when the Sommerfeld-Watson transformation is carried out on the partial-wave series for $f_{1}$ and $f_{2}$, and the differences specified in Eq. (11) are properly taken into account, the leading contributions of the Born terms to $f_{1}$ and $f_{2}$ are reproduced, as expected.

The reason that nonsense fixed poles can contribute, albeit finitely, to other amplitudes at sense points can be traced to the fact that the transformation matrix defined in Eq. (1), which connects the kinematic 
singularity-free helicity amplitudes to the usual invariant amplitudes which satisfy the Mandelstam representation, is $z$-dependent. Thus, if the "Mandelstam" amplitudes $G_{i}$ satisfy fixed- $t$ dispersion relations with no subtractions, the fixed- $t$ dispersion relations for the helicity amplitudes $f_{i}$ will generally require subtractions. These subtractions are not arbitrary, however, for the $G_{i}$ have no such arbitrariness. As we have seen, these pieces of the $f_{i}$ can be calculated explicitly from the continued partial-wave amplitudes.

It follows from the above discussion that the current procedure of Reggeizing helicity amplitudes which are free of kinematic singularities must be amended slightly; not only must provision be made for including the allowed fixed poles at nonsense points, but also for including the subsidiary contribution of these poles to amplitudes for which the point is sense.

\section{DISCUSSION}

We have seen that the Born approximation simply and successfully accounts for the $p n$ and $p \bar{p}$ chargeexchange data near $t=0$, quite in analogy with the success of the electric Born approximation in $\pi^{+}$photoproduction. At $t=0$ the dominant contribution to the differential cross section is the $u$-channel pion Born term.

We are not proposing, however, the unrestricted use of the Born approximation in the high-energy domain. There are, we believe, two features of the present problem which allow the $u$-channel pion Born term to survive more or less intact and dominate over other contributions to the amplitudes. First, there is the evasive nature of ordinary trajectories which requires most of the contributions of leading trajectories to vanish in the forward direction. We cannot prove that ordinary boson trajectories never participate in (paritydoubling) conspiracy, but the present analysis lends support to such a hypothesis. In contrast, fixed poles which reflect crossed-channel singularities automatically conspire.

The second feature which is responsible for the success of the Born approximation in the case considered is that fixed poles are allowed at right-signature nonsense points of the $t$-channel $(N \bar{N} \rightarrow N \bar{N})$ continued partial-wave amplitudes. The $u$-channel pion Born term, by itself, is consistent with an interpretation in terms of these fixed poles. We have seen that this is so provided that proper account is taken of the subsidiary contributions of these nonsense poles to amplitudes for which the corresponding point is sense.

There are cases where it appears that the Born terms might have an important effect but where they cannot. An illustrative example, because it is similar to the one considered above, is the $s$-channel reaction $p \bar{p} \rightarrow \bar{p} p$, i.e., double charge-exchange scattering. In terms of the $t$-channel $(p p \rightarrow p p)$ amplitudes, the Born contributions are given by

$s$-channel $\pi^{0}: \quad G_{1}=G_{2}=-G_{3}=-G_{4}=G_{5}=\frac{-\frac{1}{2}\left(g^{2} / 4 \pi\right)}{\left(\mu^{2}-s\right)}$,
$u$-channel $\pi^{0}: \quad G_{1}=-G_{2}=-G_{3}=G_{4}=G_{5}=\frac{-\frac{1}{2}\left(g^{2} / 4 \pi\right)}{\left(\mu^{2}-u\right)}$.

The cross section in the Born approximation is essentially independent of $t$, for small $t$ and large $s$, and is given by

$$
(d \sigma / d t)_{\mathrm{Born}}=230 \mathrm{mb} / P_{L}{ }^{2},
$$

or three times that for $p n$ charge-exchange scattering. In this case, however, we do not expect the Born approximation to have any meaning at high energy because fixed poles are forbidden by unitarity in the $J$ plane of the $t$-channel $(p p \rightarrow p p) .{ }^{8}$ The reason that fixed poles are forbidden here is the old one: There is a range of $t$ where elastic two-body unitarity holds and the spins involved are too low for the fixed cuts to play their role. The Born prediction given above is a factor $10^{3}$ larger than the experimental cross section for $p \bar{p} \rightarrow \bar{p} p$ at $P_{L}=3 \mathrm{GeV} / c, d \sigma / d t(t \simeq 0)<0.1 \mathrm{mb} / P_{L}{ }^{2} \cdot{ }^{21}$

The examples we have considered suggest that it is possible for a Born term to be meaningful at high energies if and only if it is consistent with the contribution of allowed fixed poles. Clearly, this does not imply that only Born terms contribute to fixed poles. For example, it may be that the residues of the fixed poles which are important in $p n$ and $p \bar{p}$ charge-exchange scattering are dominated by the $u$-channel pion Born term only in the region near $t=0$. Whether or not this is so will be determined by phenomenology or by means of finite-energy sum rules. At any rate, even such a limited Born-term dominance of the residues of some allowed fixed poles provides a simple mechanism for resolving some of the puzzles now facing Regge-pole theory.

${ }^{21}$ E. Escoubes et al., Phys. Letters 5, 132 (1963). 